(A us dem physiologischen Laboratorium in Zürich.)

\title{
Ueber das Verhalten der optischen Constanten des Muskels bei der Erregung, der Dehnung und der Contraction.
}

\author{
Von
}

\section{Hermann.}

Eine vor Kurzem erschienene Mittheilung von Valentin ${ }^{1}$ ) veranlasst mich, eine schon im Herbst 1879 angestellte Untersachung kurz zu veröffentlichen, welche ich, wegen ihrer überwiegend negativen Ergebnisse, ursprïnglich erst bei einem gelegentlichen Anlasse mitzutheilen gedachte. Den Anlass zu dieser Untersuchung gab mir eine Reihe von Ueberlegungen, welche ich in meinem Handbuche der allgemeinen Muskelphysik entwiekelt habe, und welche mich zu der Vermuthung führten, dass die Anisotropie des Muskels bei der Contraction abnehme ${ }^{2}$ ). Diese Vermuthung zu bestätigen, gelang mir bei der Untersuchung, wie man sehen wird, nicht, während Valentin soeben ein positives Resultat veröffentlicht.

Auf den ersten Blick könnte es scheinen als sei die vorliegende Frage schon durch Brücke erledigt, welcher angiebt, dass die optischen Constanten des Muskels sich durch die Contraction nicht ändern ${ }^{3}$ ). Allein beim Studium der Brü cke'schen Abhandlung sieht man, dass B rü cke nicht alle vorliegenden Möglichkeiten beritcksichtigt hat.

1) Dies Archiv XXI. S. 307.

2) Handbuch der Physiologie Bd. I. Theil 1. S. 248, $253 \mathrm{f}$.

3) Untersuchungen über den Bau der Muskelfasern mit Hülfe des polarisirten Lichtes. Wien 1858. S. $13 \mathrm{ff}$. Vgl. ferner Brü cke in Stricker's Handbuch der Lehre von den Geweben S. 174, und in seinen Vorlesungen über Physiologie 2. Aufl. I. S. 476. Valentin erwähnt die Brücke'schen Angaben nicht, und führt sogar den von Brücke gegebenen Beweis der positiv einaxigen Beschaffenheit des Muskels von Neuem. 
Ueb. d. Verhalten d. optischen Constanten d. Muskels b. d. Erregung etc. 241

Wenn nämlich ïberhaupt eine Veränderung der optischen Constanten stattfindet, so sind zwei Möglichkeiten vorhanden. Erstens die Veränderung ist an den Process der Erregung gebunden, findet also auch statt, wenn die Formveränderung verhindert wird, und wird in diesem Falle am reinsten und sichersten zur Anschauung kommen. So fasste Brücke die Frage auf; indem er den Muskel möglichst an der Formveränderung zu verhindern suchte, überzengte er sich, dass diejenigen Theile des Präparats, an welchen dies gelingt, ibre Farbe nicht ändern, während diejenigen, welche sich verkürzen und verdicken, eine Zanahme, diejenigen, welche durch Dehnung sich verdünnen, eine Abnahme des Gangunterschiedes, am Steigen, resp. Fallen der Farbe erkennbar, zeigen. Hieraus schloss Brücke, dass die Erregung die optischen Constanten unverändert lässt, ein Schluss, der aber nur für den Fall der verhinderten Contraction zulässig ist.

Die zweite Möglichkeit ist nämlich die, dass grade die Formveränderung mit einer Aenderung der optischen Constanten verbunden wäre, welche also bei Erregung mit behinderter Formveränderung ausbliebe. Um auch diese Möglichkeit zu erreichen, müssten bei stattfindender Verkürzung und Verdickung die Aenderungen des Gangunterschiedes festgestellt, und nachgesehen werden, ob die Gangunterschiede den Dicken proportional, oder in anderem Verhältniss sich ändern. Nur in ersterem Falle wäre auf Unveränderlichkeit der Constanten zu schliessen. Solche Messungen aber, welche tibrigens auf enorme Schwierigkeiten stossen würden, hat Brï cke nicht angestellt.

Man sieht also, dass die Brïek e'sche Arbeit die vorliegende Frage noch nicht endguiltig erledigt, so dass es gerechtfertigt war, neue Untersuchungen dariber anzustellen.

Die erste Frage, ob die Erregung an sich, ohne Formveränderung, die optischen Constanten ändert, ist schon durch Brücke so weit beantwortet, als es die unmittelbare Beobachtung gestattet. Theile des Präparates, welche weder ihre Form noch ihre Lage ändern, behalten auch ihre Farbe. An ihrer Theilnahme an der Erregung kann wohl kein Zweifel sein, da das ganze Präparat von Inductionsströmen durchflossen ist. Ich habe die einschlägigen Versuche Brücke's sehr häufig wiederholt, und den- 
selben Eindruck wie er gowonnen, sobald günstige Fälle vorkamen. Es ist nämlich, wie Briicke schon angiebt, gar nicht daran zu denken, einen Muskel so fest in Glas einzuschliessen, dass jede Verlagerung bei der Reizung und somit jede Farbenänderung durch Dicken-, Azimuth - oder Neigungsänderung ausbleibt. Wo aber ein Faserstick ganz in scheinbarer Ruhe bleibt, ist auch keine Farbenänderung zu constatiren.

Für die Unveränderlichkeit der optischen Constanten dureh blosse Erregung ohne Formveränderung spricht auch die leicht zu constatirende Thatsache, dass die Farben bei der Reizung absolut unverändert bleiben, wenn der Muskel durch Ermüdung soeben seine Contractilität verloren hat. In solchen Muskeln finden bekanntlich noch galvanische Erregungserscheinungen statt; diese also sind jedenfalls ohne Einfluss auf die optischen Constanten.

Sehr bemerkenswerth ist auch, dass, wie ich gefunden habe, der Nerv, in welchem die Erregung ohne Formveränderung abläuft, selbst bei starker Reizung sein optisches Verhalten nicht im Mindesten ändert.

Das Problem lässt sich aber noch auf einem Umwege in Angriff nehmen, der wenigstens iiber Eine Frage mit voller Sicherheit experimentell zu entscheiden gestattet. Ist nämlich eine mit der Erregung an sich verbundene und von der Formveränderung unabhängige Aenderung der optischen Constanten vorhanden, so ist es nach Analogie anderer unmittelbarer Erregungserscheinungen, z. B. der galvanischen, wahrscheinlich, dass diese Veränderung in einer gewissen Curve rasch ablätuft, und bei intermittirender Reizung des Muskels sich wie der Actionsstrom phasisch wiederholt. Die intermittirende Reizung gestattet aber eine Art mechanischen Ruhezustands des Muskels herzustellen, da in ruhigem Tetanus Dicke und Lage der Fasern sich nicht, oder wenigstens nur langsam verändern. Erhält man also einen Mnskel durch intermittirende Reizung in ruhigem Tetanus, lässt aber das Licht jedesmal nur in einer bestimmten Phase der Reizung zum Auge $\mathrm{zu}$, vergleicht man ferner die erscheinende Farbe mit der in anderer Phase oder bei continuirlichem Lichte erscheinenden Farbe, so ist in den beiden Vergleichsbeobachtungen an Gestalt und Lage der Fasern Nichts geändert, und eine etwaige phasisehe Aenderung der optischen Constanten muss sich rein und mit absoluter Sicherheit herausstellen. 
Ueb. d. Verhalten d. optischen Constanten d. Muskels b. d. Erregung etc. 243

Den angewandten Apparat ${ }^{1}$ ) will ich ,Ph asitom ${ }^{\prime \prime}$ nennen. weil er, analog dem Bernst ein'schen Rheotom, einzelne Phasen auszuschneiden gestattet. Er besteht aus einer soliden in Spitzen gehenden horizontalen Axe mit kleinem Wirtel, welche durch einen Wassermotor in sehr rasche Rotation versetzt wird. Auf die Axe sind zwei Scheiben von (geschwärztem) Messingblech ohne Isolation aufgesetzt. Die eine A, von $40 \mathrm{~mm}$ Durchmesser, ist fest, und trägt an ihrem Rande eine kleine radial gestellte Drahtgabel, welche bei jedem Umgang einmal die Menisken zweier Quecksilberröhrehen streift und dadurch den primären Kreis des erregenden Inductionsapparats vorübergehend schliesst. Die beiden Quecksilberröhrchen sind durch Kautschukschläuche mit Niveautrichtern verbunden, so dass auch bei Wegspritzen des Quecksilbers (welches übrigens bei Beobachtung aller Vorsichtsmassregeln und bei richtiger Gestalt und Einstellung der Drahtenden nicht stattfindet) die Menisken sich immer von selbst wieder einstellen; die Kautschukschläuche müssen durch Quetschhähne bis auf minimales Lumen verengt sein, sonst geräth das Quecksilber in Schwingungen. Die zweite Scheibe B, von $80 \mathrm{~mm}$ Durchmesser, besteht aus zwei Blättern, deren jedes am Rande einen Ausschnitt von $90^{\circ}$ und $18 \mathrm{~mm}$ Tiefe besitzt; beide Blätter sind zwischen zwei auf der Axe befindlichen dicken Messingscheiben eingeklemmt, welche mittels einer Schraubenmutter gegen einander gepresst werden. Dureh Verstellung der beiden Blätter, gegen einander und in ihrer Lage zur festen Scheibe A, lässt sich dem Ausschnitt jede beliebige Grösse zwischen $0^{\circ}$ and $90^{\circ}$, und ferner jede Phasenstellung gegen den Contactpunct der Seheibe A ertheilen.

Ein Hartnack'sches Polarisationsmicroscop, dessen Spiegel entfernt ist, ist mit horizontal umgelegtem Tubus auf dem Stativ des Phasitoms so befestigt, dass der Aussehnitt der Scheibe B, welcher gewöhnlich eine Breite von $30^{\circ}$ hatte, hart vor dem unter dem Objecttisch befindlichen Polariseür vorbeigeht, also bei jeder Umdrehung nur einmal während kurzer Zeit das Licht zum Microscop zulässt; als Lichtquelle dient eine Gaslampe mit Milchglascylinder. Durch eine leichte Seitenbewegung, welche bei der Aufstellung vorgesehen ist, kann das Microscop jeden Augenblick

1) Die wesentlichen Theile des Apparates besass ich schon von einer früheren, nicht publicirten (thermoelectrischen) Untersuchung her. 
so verstellt werden, dass es am Rande der Scheibe B vorbeivisirt, und doch noch (jetzt permanent) beleuchtet wird. So kann in rascher Abwechseling anf phasische und auf permanente Beobachtung eingestellt werden.

Der Apparat hat bei solider Aufstellung einen trotz sehr rascher Rotation ungemein ruhigen Gang. Zur Bestimmung der Umdrehungsgeschwindigkeit ist in den Kreis der primären Spirale ein Signal Deprez eingeschaltet, das man einige Secunden lang auf einem B altz ar'schen Cylinder schreiben lässt. Gewöhnlich machte das Phasitom 18-20 Umdrehungen in der Secunde, was zum Tetanisiren hinreichte. Bei $30^{\circ}$ Scheibenausschnitt beträgt die Beleuchtungsphase etwa $1 / 240$ Secunde.

Das Präparat bestand in einem Sartorius, der auf einem $0 b-$ jectträger zwischen zwei mit ihm gleich dicken aufgekitteten Glasleistchen und einem Deckglas fest eingezwängt war. Auf das Deckglas kam noch eine dickere Glasplatte mit kreisförmigem Durchlass für das Objectiv des Microscops; diese Platte wurde durch die federnden Objecthalter des Tisches fest aufgepresst gehalten. Der Objectträger besass zwei Stanniolstreifen, welche bis unter die Enden des Muskels reichten und als Electroden dienten. Die Behandlung des Polarisationsapparates geschah nach den bekannten Regeln; bei der Dicke des Muskels kann man meist ohne Zuhülfenahme eines Glimmerblattes auf geeignete Farben einstellen; nur mässige Vergrösserungen wurden angewandt.

Beim Tetanisiren sieht man an dem so eingezwängten Muskel natürlich trotzdem Lage- und Farbenänderungen. Stellt man nun aber rasch abwechselnd während des Ganges des Apparates auf phasisehe und auf permanente Beleuchtung ein, so beobachtet man nicht den mindesten Unterschied in der Farbe; nar die Helligkeit ist natürlich in beiden Fällen verschieden. Es ist für das Resultat völlig gleichgültig, ob man den Ausschnitt der Scheibe B gross oder klein macht, und auf welche Phase man ihn einstellt, ob auf den Moment der Reizung und die unmittelbar folgende Periode (Latenzstadium), oder auf spätere Stadien nach der Reizung.

Diese Versuche beweisen mit grösster Sicherheit, dass eine rasch vorübergehende Wirkung jeder Reizung auf die optischen Constanten nicht stattfindet; denn sonst müsste in der entsprechenden Phase die Farbe eine andere sein als bei permanenter Beleuch- 
Ueb. d. Verhalten d. optischen Constanten d. Muskels b. d. Erregung etc. 245

tung, welche die Mischung aller optischen Zustände während einer ganzen Reizperiode von $1 / 20$ Secunde darstellt.

Somit darf es als sicher festgestellt gelten, dass die Erregung an sich, ohne Formveränderung, keinen Einfluss anf die optischen Constanten des Muskels besitzt.

Mit viel grösseren Schwierigkeiten aber hat die Entscheidung der Frage zu kämpfen, ob die bei der Erregung eintretende Formveränderung einen Einfluss auf die optischen Constanten ausiibt. Der seheinbar einfache Weg hierzu ist schon oben (S. 241) angegeben worden; es fragt sich: sind die bei ungehinderter Verkürzung zu den bestehenden hinzukommenden Ganguntersehiede den Verdickungen proportional oder nicht? Aber selbst wenn jede Lageveränderung in Bezug auf Azimuth und Neigung, jede Aenderung in der Lage der übereinandergeschichteten Fasern u. dgl. ausgeschlossen wäre ${ }^{1}$ ), so wäre es weder leicht die Gangunterschiede, noch die Verdickungen exact zu messen. Und endlich ist der Verkürzungszustand des Muskels nie so lange stationär, dass zu genauen Messungen Zeit bliebe.

Bei dieser Sachlage schien es mir angemessen, zunächst eine Vorfrage zu bearbeiten, deren Schwierigkeiten minder unüberwindlich sind. Wenn die active Formveränderung des Muskels die optischen Constanten ändert, und zwar nicht sowohl in Folge der Erregung, sondern - die einzige noch uibrigbleibende Möglichkeit - in Folge der Formveränderung selbst, so ist es nicht unwahrscheinlich, dass auch passive Formveränderungen die Constanten ändern. Passive Veränderungen lassen sich aber mit viel grösserer Ruhe und Sicherheit bewerkstelligen und unterhalten.

Ich untersuchte daher das optische Verhalten des Muskels bei der Dehnung. Diese Frage bietet übrigens auch ein selbstständiges physiologisches Interesse. Bekanntlich nehmen alle Theile des Muskels bei der Dehnung gleichmässig an der Formveränderung Theil. Schon Ed. Weber verglich die Muskelfaser in dieser

1) Es ist klar, dass in jeden zur Platte senkrechten Längsschnitt des Muskels eine Anzahl Fugen fallen, und dass die Zahl derselben durch Verlagerung sich ändern kann. 
Hinsicht mit einem mit Querstreifen bemalten Kautschukfaden ${ }^{1}$ ). Es hat also grosses Interesse, wie sich die optischen Constanten der Sarcous elements bei deren passiver Gestaltveränderung verhalten.

$\mathrm{Zu}$ den Dehnungsversuchen benutzte ich einen den alten grossen Schieck'schen Microscopen beigegebenen Apparat. Das Exemplar meines Laboratoriums enthält ausser dem grossen micrometrischen Objecttisch noch eine kleinere Vorrichtung, die eine Verschiebung in zwei Richtungen durch Micrometerschrauben gestattet; von dieser wurde die eine der drei Platten sammt ihrer Schraube entfernt, so dass nur Eine micrometrische Schlittenverschiebung übrig blieb; zwei den beiden Messingplatten aufgekittete Korkstücke von passender Form und Höhe dienten zur Befestigung der Muskelenden mittels kleiner Nadeln. Eine hinzugeftigte Theilung gestattete die gegenseitige Verschiebung beider Platten abzulesen. Die Vorrichtung wurde so auf den Objecttisch des Hartnack'schen Polarisationsmicroscopes gelegt, dass die Faserrichtung mit den Polarisationsebenen der gekreuzten Nicols einen Winkel von $45^{\circ}$ bildete.

Die Präparate bestanden aus dünnen, nur wenige Fasern enthaltenden Bündeln, welche den Oberschenkelmuskeln des Frosches entnommen waren.

Vor Allem zeigt sich, dass mit der Dehnung ausnahmslos ein Fallen der Farben, d. h. eine Abnahme des Ganguntersehiedes eintritt, wie sie in Folge der Verdünnung des Muskelbündels auch von vornherein zu erwarten war. Es fragt sich nun weiter: entspricht die Abnahme des Gangunterschiedes der Verdünnung? Ist in der Grösse

$$
\left(n^{4}-n\right) D
$$

welcher der Gangunterschied bei gegebener Lage und Wellenlänge proportional ist ( $\mathrm{n}^{\prime}$ ist der extraordinäre, $\mathrm{n}$ der ordinäre Index, D die Schichtdicke), durch die Dehnung nur D, oder anch $n^{\prime}-n$ geändert? Im ersteren Falle muss der Gangunterschied der Dicke stets proportional bleiben.

Aus einer von Billet nach Untersuchungen von Wertheim gegebenen Tabelle ${ }^{2}$ ) lässt sich für zwei beobachtete Farben das

1) Wagner's Handwörterbuch der Physiologie III. 2. S. 65.

2) Billet, Traité d'optique physique I. p. 490, II. p. 639; die Tabelle 
Verhältniss der entsprechenden Gangunterschiede entnehmen, obgleich die absoluten Gangunterschiede daselbst nur für einen Weg in Luft angegeben sind. Die Uebersetzung der Farben in Zahlen kann freilich nur ziemlich angenau geschehen, indess reicht sie ans für den hier vorliegenden Zweck. Die Verdünnung lässt sich aus der am Apparate abgelesenen Verlängerung entnehmen, das Verhältniss der Dicken muss, bei erhaltener sonstiger Form, umgekehrt proportional sein der Quadratwurzel aus dem Verhältniss der Längen.

. In eịner grossen Zahl von Versuchen wurden nun die Dehnungen und die Farbenveränderungen unmittelbar notirt, und nachgesehen, ob die Gangunterschiede den in angegebener Weise berechneten Dicken proportional sind. Es zeigte sich indess, dass die Gangunterschiede bei der Dehnung beträchtlich stärker abnehmen, als der Proportionalität mit der berechneten Dicke entspricht. Entweder also ändern sich die optischen Constanten in dem Sinne, dass $\mathrm{n}^{\prime}-\mathbf{n}$ bei der Dehnung geringer wird (der Muskel der Isotropie sich nähert), oder die Dicken sind geringer als sie angenommen werden. Das Erstere ist nun höchst unwahrscheinlich; wenn die Dehnung die Constanten. ändert, so wäre jedenfalls im Gegentheil nach Analogie der bekannten Erscheinungen an gespannten Gläsern eine Zunahme der positiven Anisotropie zu erwarten, da die Theilchen in der Richtung der Axe weiter auseinander rücken, senkrecht dazn aber sich nähern. Der Grund der beobachteten Erscheinung liegt vielmehr darin, dass, obgleich die einzelne Faser ohne Zweifel bei der Dehnung cylindrisch bleibt, ihre Dicke also der Rechnung entspricht, das Bündel sich bei der Dehnung abplattet, also dünner ist, als berechnet. Der Versuch müsste also, um beweiskräftig zu șein, mit einzelnen Fasern angestellt werden; es gelingt aber nicht, solche zu dehnen ohne sie zu zerreissen.

Ich schlug nunmehr einen anderen Weg ein, nämlich die Vergleichung der Farbe gedehnter und ungedehnter Muskelschichten von gleicher Dicke. Anfangs wurden gleichzeitig zwei Muskeln, ein gedehnter und ein ungedehnter, neben einander in das 
Gesichtsfeld gebracht; dieselben waren zwischen zwei Korkplatten ausgespannt, und befanden sich mit dem untersuchten Theil zwischen zwei Spiegelglasplatten eingepresst, deren Abstand durch zwei dünne Glasstreifen fixirt war; ich unterlasse die specielle Beschreibung der benutzten Vorrichtung. Der Zufall wollte es dass die ersten Beobachtungen mit grösster Regelmässigkeit einen grösseren Gangunterschied des gedehnten Muskels ergaben, also im Sinne einer Zunahme der Positivität sprachen: Ich hatte nämlich, um dem gedehnten und dem nicht gedehnten Muskel schon von vorn herein annähernd gleiche Dicke zu geben, also gleichen Druck der regulirenden Glasplatten herzustellen, den zu dehnenden Muskel dicker genommen, and zu diesem den Sartorius, zum andern den Cutaneus gewählt; der gedehnte Sartorius zeigte nun in gleicher Schicht jedesmal höheren Gangunterschied als der ungedehnte Cutaneus. Als ich aber endlich zu Controllversuchen schritt, ergab sich, dass auch bei gleichem Dehnungsgrade die Anisotropie des Sartorius stärker ist als die des Cutaneus, und iiberhaupt in dieser Hinsicht die verschiedenen Muskeln des gleichen Thieres stets Differenzen zeigen. Dieselben können sowohl von der versehiedenen Entwicklung der isotropen Zwischengewebe (Perimysien, Sarcolemme), als von dem verschiedenen Verhältnisse der isotropen und anisotropen Theile im Muskelquerschnitt herrühren, ganz abgesehen von der Möglichkeit verschiedener Constanten der anisotropen Theile selbst.

Um nun diese Fehlerquelle gänzlich zu eliminiren, verglich ich endlich die Farbe des gleichen Muskels in verschiedenen Dehnungsstadien bei stets gleicher, durch Druck zwischen Glasplatten hergestellter Schichtdicke. Ich benutzte dazu den oben erwähnten Apparat mit feiner Schraubenverschiebung, welcher nunmehr eine kleine Abänderung erhielt. Zwischen beiden Korkstïcken wurde nämlich eine Spiegelglasplatte auf die obere durchbohrte Messingplatte gelegt; die Oberfäche der Glasplatte war genau in gleicher Ebene mit den Korkflächen; der Muskel wurde also über die Glasplatte hinweg gespannt. Die zweite, aufgepresste Spiegelglasplatte wurde wiederum durch Glaslamellen in constantem Abstande erhalten und selbstverständlich während der Verschraubung selbst jedesmal entfernt; nach jeder Dehnung wurden die Glasplatten so verschoben, dass sie jedesmal denselben Theil des Muskels zwischen sich fassten. 
Ueb. d. Verhalten d. optischen Constanten d. Muskels b. d. Erregung etc. 249

Das constante Resultat dieser Versuche war: die Farbe wird durch die Dehnung nicht merklich verändert. Die Dehnung ist also ohne nachweisbaren Einfluss auf die optischen Constanten der anisotropen Gebilde des Muskels.

Zu den Versuchen selbst will ich noch bemerken, was übrigens Jeder, der auf diesem Gebiete schon gearbeitet hat, wissen wird, dass was hier mit Farbe bezeichnet ist, eben nur die vorherrschende Farbe im Gesichtsfelde ist. Zwischen den Muskelfasern erseheinen natürlich immer Streifen von anderer Farbe. In einem sorgfältig hergestellten Präparate mit schön parallel liegenden Fasern, das den Raum zwischen zwei Glasplatten genau ausfüllt, ist aber, und zwar aus naheliegendem Grunde gerade bei dickeren Schichten, wie der Sartorius, die allgemeine Färbung wenigstens so gleichartig, dass man sehr gut Aenderungen derselben constatiren kann. Wer nun bei der Wiederholung der Versuche kleine Aenderungen durch die Dehnung: zu erkennen glaubt, wird bei genügender Vermehrung der Beobachtungen sich überzengen, dass Täuschungen durch nicht genaue Fixation (so dass die Dehnung Verlagerung bewirkt) u. dgl. vorlagen, und dass ïberhaupt bei diesen an Fehlerquellen so reichen Versuchen jede zu minutiöse und jede nicht ganz regelmässig auftretende Veränderung als nicht constatirt gelten muss. Ich urgire dies namentlich deshalb, weil ich selber längere Zeit zweifelhaft war, ob nicht eine sehr geringe Erhöhung des Gangunterschiedes in den gedehnten Muskeln erkennbar ist.

Die Erhaltung der optischen Constanten bei der Dehnung ist so merkwürdig, dass mancher vorziehen wird, trotzdem eine geringe Aenderung anzunehmen, welche jedoch innerhalb der Fehlergrenzen unserer Beobachtung liegt. Auch ich war sehr davon überrascht, denn Brücke hatte zwar jene Erhaltung schon ausgesprochen, aber nur constatirt, dass die Gangunterschiede durch Dehnuug abnehmen, also bis auf Weiteres kein Grund zur Annahme einer Constantenänderung vorliegt, welche trotzdem vorhanden sein konnte.

Gehen wir nun endlich zur Zusammenziehung des Muskels über, so ist die Erwartung, dass dieselbe mit einer Aenderung der optischen Constanten verbunden sei, nunmehr bedeutend vermindert, da nachgewiesen ist, dass weder die passive Formver- 
änderung, noch die Erregung ohne Formveränderung, die Constanten ändert.

Zu den Versuchen benutzte ich dünne Faserbündel, welche aus den tieferen Oberschenkelmuskeln der Kröte dureh Zug mit einer feinen Pincette entnommen und in sorgfältig gestreckter Lage auf den Reizobjectträger unter $45^{\circ}$ gegen die gekreuzten Nicols, ohne Deckglas, gebracht wurden. Selbst sehr dünne Bündel sind gut erregbar. Bei den Contractionen habe ich nun immer nur Steigen, nie Sinken der Farbe beobachtet, also lediglich die Angabe Brücke's bestätigt. Die Zunahme des Gangunterschiedes bei der Verdickung ist nun freilich noch lange kein Beweis, dass die Constanten unverändert bleiben. Es gelang mir aber nicht, diesen Beweis, etwa wie für die Dehnung, zu führen. Die Schwierigkeiten exacter Beobachtung sind hier, wo Krümmungen unvermeidlich mit ins Spiel kommen, enorm viel grösser. Eine Abnahme der positiven Anisotropie wäre freilich leicht zu constatiren, wenn sie den Einfluss der Verdickung uibercompensirte, also Sinken der Farbe oder gar völlige Dunkelheit (Isotropie) hervorbrächte; dies habe ich aber niemals constatiren können.

Valentin ist nun, nach seiner Eingangs citirten Mittheilung; glücklieher gewesen. Es gelang ihm nicht allein, einzelne isolirte Fasern unter dem Microscop zu tetanisiren (was ich nie erreicht habe), sondern sogar während des Tetanus die Faser durch alle Azimuthe zu drehen, wobei er sich bestimmt überzeugt haben will, dass die Anisotropie bis zu Null abnimmt. Die oben behandelte Frage, wie sich der Muskel bei verhinderter Verkürzung verhalte, wird von Valentin nicht berïhrt.

Ich habe nach dem Erscheinen der Valentin'schen Arbeit meine Versuche sämmtlich wiederholt, und einige oben schon mit berücksichtigte Vervollkommnungen eingeführt, konnte aber nur die früher gewonnenen Resultate bestätigen. Jedenfalls hätte mir ein vollständiges Schwinden der Anisotropie bei der kräftigen Contraction meiner Präparate unmöglich entgehen können.

Das Resultat meiner Versuche lässt sich kurz folgendermassen formuliren: Dehnung bewirkt keine nachweisbare Aenderung der optischen Constanten; ebensowenig die Erregung, wenn die Formveränderung verhindert ist; auch findet keine phasische Aenderung der Constanten in irgend einem Stadium der Erregung Statt. Bei zugelassener Contraction des Muskels ist eine Aende- 
Ueb. d. Verhalten d. optischen Constanten d. Muskels b. d. Erregung etc. 251

rung der Constanten nicht nachzuweisen, wenn auch durch die hier sehr unvollkommenen Versuche nicht positiv ausgeschlossen. Der Nerv ändert sein optisches Verhalten bei der Erregung nicht.

Die von Brü cke gemachte Annahme, dass die eigentlichen doppeltbrechenden Elemente (Disdiaclasten) die Gestaltveränderungen des Muskels nicht mitmachen, und dass ihre Anzahl in einem Querdurchmesser der Faser allen Aenderungen dieses Durchmessers proportional sich ändert, wird durch die vorstehenden Erfahrungen bestätigt. Die Brücke'sche Ausdrucksweise ${ }^{1}$ ), dass bei der Contraction , ähnlich wie bei einer Compagnie Soldaten versehiedene Breiten und Tiefen der Aufstellung durch Ortsveränderung der einzelnen Individuen erzielt werden", enthält bei näherem Zusehen nichts was nicht auch auf die Dehnung passte; denn es ist kein Grund vorhanden, jene Ortsveränderung als etwas Actives, etwa als die Ursache der Contraction, aufzufassen. Ein mit spindelförmigen längsgerichteten Glasstückehen dicht erfuillter Kautschnkschlauch würde, wenn die Reibung zwischen denselben sehr vermindert wäre, bei der Dehnung und Wiedernachlassung ganz ähnliche Verlagerung der Glasspindeln zeigen, wie sie für die Disdiaclasten in der Muskelfaser oder im Sarcous element anzunehmen sind.

Ob die Anisotropie des Muskels eine wesentliche functionelle, und nicht vielmehr eine nur morphologische Bedeutung hat, muss hiernach höchst zweifelhaft erscheinen; jedenfalls erledigen sich zunächst die in meiner allgemeinen Muskelphysik, Seite 248 und 254, ausgeführten Betrachtungen.

1) Stricker's Handbuch etc. S. 174. 